\title{
Bleeding sarcoma of the aorta mimicking a symptomatic aneurysm
}

Bart P. Van Putte, MD, PhD, ${ }^{a}$ Thomas L. Bollen, MD, ${ }^{b}$ and Marc A. A. M. Schepens, MD, PhD, ${ }^{a}$ Nieuwegein, The Netherlands

丹 Supplemental material is available online.

\section{Clinical Summary}

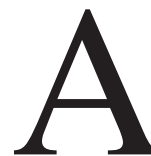

59-year-old man was admitted to our hospital with a history of coronary artery bypass grafting 2 years before. On admission, he reported having exerciseinduced dyspnea (New York Heart Association class III). Physical examination revealed decreased breath sounds at the left lower pulmonary fields and blood analysis showed a hemoglobin level of $7.7 \mathrm{mg} / \mathrm{dL}$. Contrast-enhanced computed tomography (CT) (Figure 1,B) and magnetic resonance imaging (MRI) (Figure 1, A) of the aorta showed an aneurysm of $9.7 \times 8.0 \mathrm{~cm}$ located on the left lateral aspect of the lower descending aorta. The presence of fluid in the left pleural space was suggestive of a leaking aortic aneurysm. Therefore, the patient was urgently treated with two aortic endoprostheses (Valiant $30 \times 30$ and $38 \times$ $38 \mathrm{~mm}$; Medtronic, Inc, Minneapolis, Minn) successfully inserted under radiographic control.

After 3 months, the patient was readmitted with exerciseinduced dyspnea (New York Heart Association class III), decreased exercise tolerance, weight loss of $7 \mathrm{~kg}$ since discharge, and pain in the upper abdomen. Physical examination showed decreased breath sounds at the left thoracic side. Hemoglobin concentration had decreased by $1.9 \mathrm{mg} / \mathrm{dL}$ in 3 months. Under suspicion of endoleakage, contrast-enhanced CT (Figure E1) and MRI (T1 after gadolinium) (Figure 2, A) of the aorta showed a heterogeneous and lobulated mass of $12.7 \times 9.4 \mathrm{~cm}$ located in the lateral aspect of the aortic wall bulging into the left pleural space, but there was no sign of contrast extravasation suggestive of endoleakage. On the basis of the second CT, a tumor was suspected and further imaging techniques (ultrasonography, MRI, and positron emission tomography [PET] scan) strengthened this diagnosis. PET scan (Figure 2,B) revealed high uptake in the aortic wall,

From the Departments of Cardiothoracic Surgery ${ }^{\mathrm{a}}$ and Radiology, ${ }^{\mathrm{b}}$ St Antonius Hospital, Nieuwegein, The Netherlands.

Received for publication Nov 10, 2006; revisions received Nov 29, 2006; accepted for publication Dec 7, 2006.

Address for reprints: B. P. van Putte, MD, PhD, Department of Cardiothoracic Surgery, St Antonius Hospital, Koekoekslaan 1, Nieuwegein, The Netherlands (E-mail: bvanputte@yahoo.com).

J Thorac Cardiovasc Surg 2007;133:1643-4

$0022-5223 / \$ 32.00$

Copyright () 2007 by The American Association for Thoracic Surgery doi:10.1016/j.jtcvs.2006.12.070
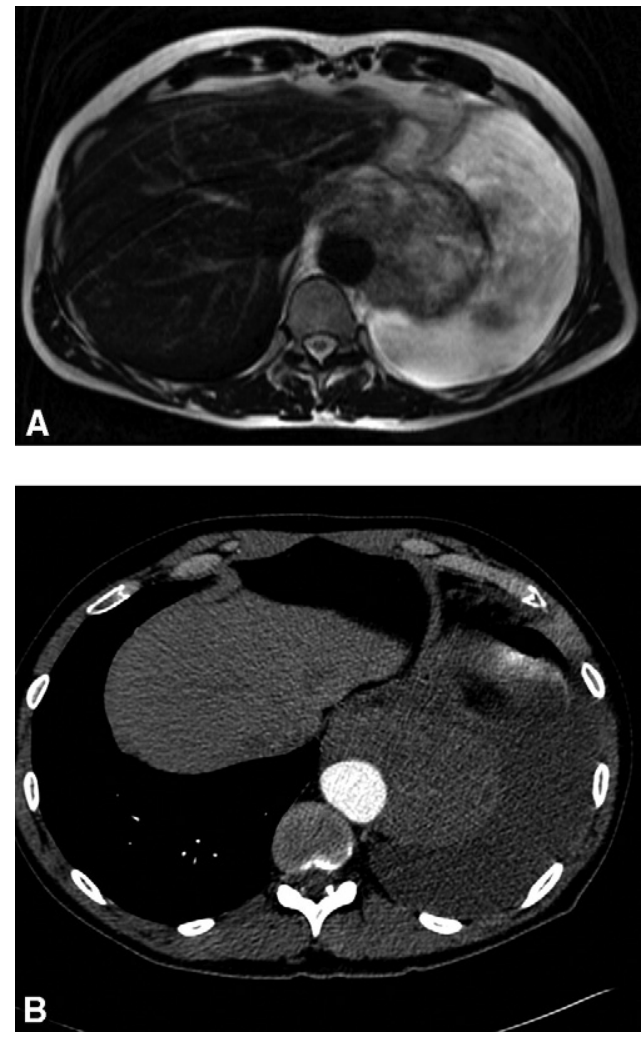

Figure 1. MRI (A) and contrast enhanced CT (B) pictures of the aorta show an aneurysm of $9.7 \times 8.0 \mathrm{~cm}$ located on the left lateral aspect of the lower descending aorta. The presence of fluid in the left pleural space was suggestive of a leaking aortic aneurysm.

which looks similar to the corresponding CT and MRI images. The high uptake in this case is an indicator of malignant disease and would not be seen in a leaking symptomatic aneurysm. Subsequently, tissue was obtained by ultrasonography guided biopsy, which revealed undifferentiated sarcoma.

The patient was operated on and left heart bypass was performed from the descending aorta to the right femoral artery. The tumor had infiltrated the left hemidiaphragm, pericardium, and left lower lobe. Therefore, the descending aorta, including the sarcoma, was resected from the seventh level up to the superior mesenteric artery, en bloc with the hemidiaphragm, part of the pericardium, left lower lobe, and lingula (Figure 2). A Dacron interposition graft was used for restoration of aortic continuity and the diaphragm and pericardium were repaired with Marlex mash and bovine pericardium, respectively. Pathologic examination of the tumor revealed pleomorphic sarcoma. 

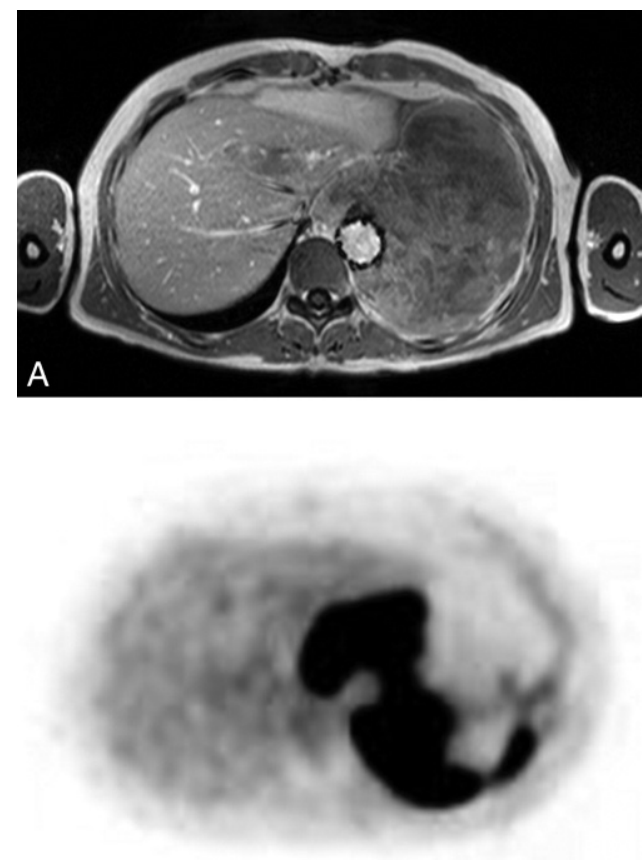

B

Figure 2. A, Three months after endoprosthetic treatment, MRI (T1 after gadolinium) of the aorta was made under suspicion of endoleakage. This scan shows a heterogeneous and lobulated mass of $12.7 \times 9.4 \mathrm{~cm}$ located in the lateral aspect of the aortic wall bulging into the left pleural space. B, Three months after endoprosthetic treatment, PET scan reveals high uptake in the aortic wall, which looks similar to the corresponding CT and MRI images. The high uptake in this case is an indicator of malignant disease and would not be seen in a leaking symptomatic aneurysm.

The postoperative course was complicated by a necrotic gall bladder, which required a cholecystectomy. The patient did not receive adjuvant radiotherapy because the large size of the radiation field would have resulted in a disproportional risk of cardiotoxicity and no response was expected from chemotherapy. After 6 months, the patient was in good condition and follow-up CT did not show evidence of recurrent disease (Figure 3 ).

\section{Discussion}

The diagnosis of aortic sarcoma is rare and is established only during or after operation or at autopsy. ${ }^{1}$ Most aortic sarcomas originate from the intimal layer and are characterized by an intraluminal growth pattern often resulting in partial aortic occlusion and embolization.

In this case, the initial CT was characterized by an intramural process originating from the lateral side of the descending aorta in combination with a large amount of pleural fluid present at the same side. Together with findings from history and physical ex-

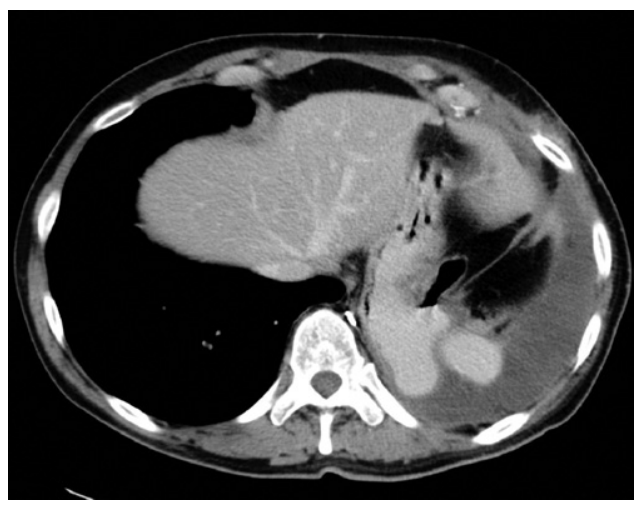

Figure 3. Six months after tumor resection, thoracic and abdominal CT was repeated to look for evidence of recurrent disease. The scan shows residual pleural fluid at the left side without any signs of pleural metastases. Furthermore, herniation of part of the stomach is depicted as a result of resection and reconstruction of the left hemidiaphragm.

amination, this presentation of an aortic sarcoma mimicked a symptomatic and leaking aortic aneurysm. Owing to the extremely low incidence of an aortic tumor, however, the diagnosis of a symptomatic aneurysm was made at initial presentation and the final diagnosis of aortic sarcoma was therefore delayed for 3 months.

During the second admission, retrospective comparison of the CT and MRI revealed an increased size of the aortic mass but no signs of endoleakage (Figure 1). Furthermore, the aortic mass was characterized by a distinct heterogeneous appearance present on the lateral side of the aorta. Furthermore, the mass was draped lobulated around the lumen of the aorta. These findings were suggestive of a tumor. The diagnosis of a tumor was strengthened by demonstration of vascularity in the mass by Doppler ultrasonography and enhancement of the mass on MRI. The malignant nature of the tumor was highly suspected as a result of the high uptake on PET scan; to our knowledge, a preoperative PET scan of an aortic sarcoma has never been described. Owing to the low incidence of aortic sarcoma, the premortem diagnosis and imaging of aortic sarcoma is highly unusual.

Although aortic sarcoma remains an exceptional disease, its presence should be considered in a case of atypical presentation of a symptomatic aortic aneurysm. Furthermore, a high degree of uncertainty is needed when interpreting imaging techniques in these patients. ${ }^{2}$

\section{References}

1. Steinberg JB, Johnson ER, Benda JA, Lanza LA. Primary leiomyosarcoma of the thoracic aorta presenting as a contained rupture. Ann Thorac Surg. 1993;56:1387-9.

2. Muehlstedt S, Mallery S, Joyce L, Van Camp J. Myxoid leiomyosarcoma of the descending thoracic aorta. J Thorac Cardiovasc Surg. 2001;121:590-2. 


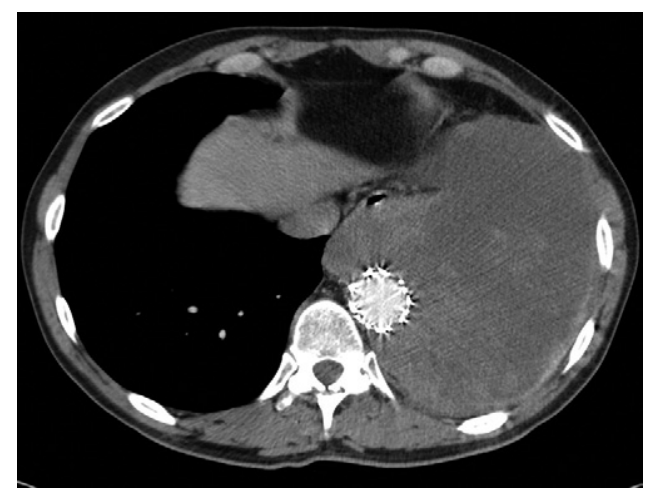

Figure E1. Three months after endoprosthetic treatment, contrastenhanced CT of the aorta was made under suspicion of endoleakage. This picture shows a heterogeneous and lobulated mass of $12.7 \times 9.4 \mathrm{~cm}$ located in the lateral aspect of the aortic wall, bulging into the left pleural space, but there is no sign of contrast extravasation suggestive of endoleakage. 